\title{
The Cost of Inappropriate Referral Pathways in Inpatient Care for Three Major Noncommunicable Diseases in Mongolia: A Registry-Based Analysis
}

Ariuntuya Tuvdendorj ( $\nabla$ t.ariunaa@gmail.com )

University of Groningen https://orcid.org/0000-0002-3847-6974

Otgonjargal Dechinkhorloo

General Authority for Health Insurance

Bayarsaikhan Dorjsuren

World Health Organization

\section{Erik Buskens}

University of Groningen: Rijksuniversiteit Groningen

\section{Talitha Feenstra}

University of Groningen: Rijksuniversiteit Groningen

Research article

Keywords: Non-communicable diseases (NCDs), pulmonary, Mongolia

Posted Date: June 30th, 2021

DOI: https://doi.org/10.21203/rs.3.rs-659195/v1

License: (c) (i) This work is licensed under a Creative Commons Attribution 4.0 International License. Read Full License

Version of Record: A version of this preprint was published at BMC Health Services Research on November 27th, 2021. See the published version at https://doi.org/10.1186/s12913-021-07281-8. 


\section{Abstract}

Background: Non-communicable diseases (NCDs) consistently pose a huge economic burden to health systems and countries in general. The aim of this study was to quantify inpatient costs associated with chronic obstructive pulmonary disease, stroke and ischemic heart disease stratified by type of referral pathway, and to investigate key factors that drive these costs.

Methods: A registry-based data analysis was performed using national public hospital inpatient records from 2016 to 2018 for 117,600 unique patients and linking patient-level inpatient health care use with hospital-specific unit cost per bed-day. These were combined to calculate the annual inpatient costs for each of the three disorders per person and per year. Generalized linear modeling was used to assess the association of inpatient costs with age, gender, location, comorbidity, treatment referral pathways and years.

Results: Across three diagnoses, the majority of patients were female. Most were over 50-60 years old, with more than half being a pensioner, typically with at least one comorbidity. About $25 \%$ of patients followed what might be considered inappropriate (unofficial) inpatient referral pathways. Mean annual inpatient costs were int $\$ 21$. These costs rose to int\$ 849 for unofficial pathways and dropped to int $\$ 677$ for official pathways. Further covariates significantly associated with high inpatient costs were location, age, gender, and comorbidity.

Conclusion: Our findings provide background information essential to develop evidence-based and costeffective interventions aimed at health promotion, prevention and service delivery. Reducing the unofficial use of inpatient care can improve efficient resource allocation in health care and prevent further escalation of inpatient costs in the future.

\section{Key Points}

- Mean inpatient costs associated with chronic obstructive pulmonary disease, stroke and ischemic heart disease were significantly higher for unofficial referral pathways compared to official referral pathways.

- Key drivers of high inpatient costs next to referral pathway were location, age, gender and comorbidity.

- Factual costs of hospital care for the NCDs considered provide insight in healthcare delivery, and the financial burden of disease for Mongolia and comparable developing countries.

\section{Introduction}

The costs associated with non-communicable diseases (NCDs) consistently pose a high economic burden on health systems and countries in general [1,2]. With an increasing prevalence of NCDs, the main challenges for health system financing in many low- and middle-income countries (LMICs) are 
ensuring financial sustainability and efficient resource allocation in health care [3]. Globally, average per capita health spending is approximately US $\$ 1100$, but in absolute terms most LMICs spend less than US\$ 130 [4]. The high pressure NCDs put on LMICs and their limited budgets may be reduced by preventing and improving the efficiency of NCD-related hospitalizations. To support policymakers, accurate information on the costs related to hospitalizations for NCDs and the factors that influence these costs is important.

One of the factors might be hospital referral pathways. Lack, either real or perceived, of quality access to or the capacity of community level hospitals may lead to inefficient treatment pathways, with direct entry into secondary or tertiary levels of care. Higher level facilities are often better equipped and employ more specialized personnel, hence self-referral tends to occur [5]. The downside may be that tertiary care hospitals become overcrowded. In response, the length of stay for stroke patients has been reduced to about six days, while some hospitals transfer patients to lower-level hospitals [6]. Previous evidence suggests that about $20-40 \%$ of resources spent on health care may be wasted due to inefficiency and unofficial service use [7]. Inefficient referral may cause unofficial service use. Hence it is relevant to specifically investigate the impact of referral pathways on treatment costs, especially for LMICs.

Mongolia is a LMIC with a population of 3.3 million $[8,9]$. The country's health infrastructure is challenged by the extremely low population density over a large territory under rapid economic transition. The health system is largely hospital-based [5]. This practice pathway emerged during the era of free access to publicly-funded hospitals. [10] Currently, NCD related inpatient care is included in the social health insurance benefits package. Service providers are paid according to a diagnosis-related groups (DRG) payment mechanism [11]. About two thirds of public health spending on NCDs in 2013 (56.1 billion MNT out of 85.2 billion MNT) was for inpatient care (hospital admission) [12]. Despite apparent unique challenges for the provision of service delivery in Mongolia, elsewhere NCDs are similarly the leading cause of death and disability, responsible for almost $80 \%$ of all deaths in 2018 [13]. In many aspects, Mongolia displays trends very similar to those previously signaled for LMICs in general: a rise in life expectancy, accompanied by an increase of NCDs [14]. Meanwhile, an increasing prevalence of lifestyle related risk factors for NCDs is observed. The need to better understand what drives hospital costs for NCDs in Mongolia, as well as in other LMIC countries is clear.

Inpatient costs for NCDs and specifically for COPD, IHD, and stroke have been shown to vary with several factors. The most studied factors that contribute to high inpatient costs in LMICs were age, gender, comorbidities, length of stay, and type of health care providers [15]. Disease-specific factors that drive inpatient costs were type of stroke in China [16] and lung function for patients with COPD in Vietnam [17], both reflecting disease severity. Disease-specific costing studies thus far were typically conducted in a single hospital and were limited by small sample size. Generalizability to countrywide costs may thus be limited $[16,18]$.

The current study adds to this literature. Using a large patient-level dataset from the national Mongolian hospital inpatient registry, in combination with data from hospital financial accounts, information beyond 
what has previously been published may be retrieved [12]. The database comprises information on patient characteristics and their utilization of inpatient care over a three-year period. In this study, inpatient costs were estimated using a health care provider perspective, while distinguishing a range of cost drivers. The aim of the study was to estimate population mean inpatient costs associated with three diagnosis-related groups (DRGs) of diseases between 2016 and 2018, and to investigate key factors that drive high inpatient costs for these 3 DRG groups in Mongolia, in particular the role of referral pathways.

\section{Methods}

\subsection{Study design}

A 'real world data' registry-based cost analysis was performed using national public hospital inpatient records for the years 2016 through 2018 in Mongolia. Two data sources were combined to calculate the population mean costs associated with hospitalization for patients with stroke, chronic obstructive pulmonary disease (COPD) and ischemic heart disease (IHD). First, micro-level hospital registry data was used to analyze the characteristics of inpatient care use across different diagnosis-related groups (DRGs). Second, hospital-specific funding source data were collected from hospital financial reports to calculate the unit cost per bed-day. Public funding sources, that is, direct government funding and health care insurance for each type of hospital, were tracked over time. Inpatient health care use was linked with cost per bed-day in order to quantify the costs per patient per year (PPPY). This was repeated for each of the three conditions. Generalized linear modeling analysis (GLM) was used to relate the PPPY to several covariates reflecting patient characteristics, type of treatment referral pathways and diagnosis.

\subsection{Data sources}

The national inpatient records are managed by the Health Development Center, which is the public authority responsible for collecting, inspecting and annual reporting of national health indicators in Mongolia. The registry uses the international classification of diseases version 10 (ICD-10). We selected the three most prevalent DRGs among the NCDs: COPD (J40-J44), IHD (I20-I25), and stroke (I60-I69).

Inpatient record data contains information on demographics (i.e., age, sex, place of residence, and education), socioeconomic characteristics (social status, working area and profession), medical condition (diagnosis, number of bed-days,) and type of health care provider. An anonymized patient identification number was used to record any comorbidity as present in a given year. Inpatient care use was estimated at patient-level rather than per admission only. Laws on transparency in Mongolia, effective as of 2015 , all state authorities, including public hospitals, are obliged to report financial information online and make it freely accessible to the public. Hospital financial reports contain information on total funding and funding sources. The extracted data were further validated through expert consultations. 


\subsection{Participants}

Our dataset consisted of all inpatient admissions in Mongolia in the years 2016 to 2018 (Fig 1). Admissions that had less than 2 bed-days were excluded. Also, due to unavailable financial information, admissions in private and specific public hospitals that provide inpatient care for specific groups including the railway, court and military sector workers rather than general population were excluded.

A total of 136,925 admission records were selected from 303 public hospitals, including 228 community (soum) hospitals, 39 inter-community hospitals (all primary level care), 16 provincial (aimag) general hospitals, 5 regional diagnostic centers, 12 district general hospitals in Ulaanbaatar (all secondary level care), and 3 general clinic hospitals (tertiary level care).

Patients living in rural and urban areas follow different inpatient care pathways which reflect geographical circumstances (Fig 2). Generally, patients living in the capital city receive inpatient care from district level hospitals [19]. Their health care provider then decides whether they need referral to a general hospital for specialized care. Similar procedures apply to patients in rural areas. Depending on the proximity of the hospital, patients in rural areas first receive inpatient care at community or intercommunity hospitals, and if they cannot be treated effectively, these hospitals refer patients to provincial or regional hospitalswhich may, if needed, further refer patients to tertiary level care (the general clinic hospitals in Ulaanbaatar). However, in practice, some patients bypass community and district hospitals and go straight to secondary or tertiary levels care, thus following unofficial referral pathways [5].

\subsection{Variables}

The outcome variable in the analysis was total hospitalization cost per patient per year. Since price levels varied over time, they were adjusted to the price level of 2018, based on the consumer price index for all three years in the final analyses. The primary covariates included in the model were gender, age, comorbidities, location of the patient, social status, treatment referral pathways and year. Sex was dichotomized (female versus male); age was categorized into ten-year age groups: 0-40, 40-50, 50-60, 6070, 70-80 and 80 and older; patient location was dichotomized (urban versus rural); and patient comorbidities were identified within the three ICD codes recorded during each hospitalization. Social status was categorized into 5 categories: formal sector salaried employee, private sector employee, herders, pensioners and others, including full-time students and children aged under 15 years. Patient referral pathways were assessed and coded as dummy variables based on each individual patient's admissions. The dummy variable indicated whether the treatment path was an official or unofficial referral by merging information on referral paths with patient location information over the one-year period.

\subsection{Costing}


Costs per patient per year were estimated by type of hospitals and diagnosis, using patient-level data for information on number of admissions and number of bed-days, and hospital financial records for hospital-level information on total budgets. To arrive at costs per patient per year, the number of bed-days for each patient and diagnosis were multiplied by the relevant unit costs per bed-day, depending on hospital type and diagnosis. An overview of hospital funding information and share of funding sources are presented in Table S1 in the supplementary material. More detailed information about the payment methods and costing estimation is also provided in the supplementary material Table S2.

\subsection{Statistical analysis}

Statistical analyses were performed in R software version 1.2.5033 by RStudio Inc, Boston US, while data preprocessing of hospital accounting information was performed in MS Excel. More detailed information related to the statistical analysis is reported in the supplementary material. Costs were estimated in Mongolian National Tugrik (2472 MNT 1 USD in 2018) in real terms at base year in 2018. Findings are reported in international dollars (int\$) by using purchasing power parity (PPP\$) exchange rates for the period 2016 to 2018 [20].

\section{Results}

Table S3 in the supplementary material summarizes the trends in inpatient care services according to the hospital levels and hospital types. A total of 136.9 thousand admissions were recorded with similar numbers in all years. Almost one third of the inpatient care was delivered by the secondary level provincial hospitals, followed by the primary level community hospitals in rural areas. The largest number of admissions was recorded for patients diagnosed with IHD, followed by admissions for stroke and COPD. Table S4 in the supplementary material presents mean costs per bed-day across different hospital types. The cost for IHD was the highest at int $\$ 80$ per day, followed by COPD (int $\mathbf{7 4}$ ) and stroke (int\$ 67). Figure 3 illustrates the mean per bed-day costs by types of hospital and years for stroke patients, and the stroke-related total number of bed-days (Fig 3). Cost per bed-day decreased over time after converting the real price to the 2018 price level and international dollars. When comparing all hospitals, the mean cost per bed-day in the primary level hospitals was the highest, while the total number of bed-days was the least in these hospitals. Similar information for COPD and IHD can be found in the supplementary material figure S2-S3.

A total of 117.6 thousand unique patients received inpatient health care. (Supplementary Table S5) The majority of the patients were female and more than half of the patients were pensioners who had at least one comorbidity. In general, male patients tended to stay in hospital longer than female patients. Patients who resided in rural areas more frequently received inpatient care due to stroke and IHD, whereas patients in urban areas were most frequently hospitalized for COPD.

In total, 14 possible patient referral pathways by diagnosis were found and their distribution varied across the diagnoses. (Supplementary Table S6) About $7-22 \%$ of the patients directly received inpatient care at tertiary level hospitals, indeed bypassing primary and secondary hospitals. 
Table 1 shows the mean inpatient costs distribution for the whole sample over the one-year period. Mean inpatient costs differed significantly according to age group, location, comorbidity, and social status when stratified by type of referral pathway. About one quarter of the patients followed unofficial referral pathways, meaning direct referral to a higher level of hospital. Overall mean inpatient costs for all three diagnoses was int 721 (sd=416) and the amounts increased from int\$ 677 for official referral pathways to int\$ 849 for unofficial referral pathways. In general, higher costs were seen for men, older age groups, herders and people living in rural areas.

Table 2 summarizes the results of the multivariate regression analysis for total inpatient costs. Annual mean inpatient costs were equivalent to int\$ 642 in the reference group (being female patients aged under 40 years old, living in urban areas, who work in the formal sector and followed an official referral path). Inpatient costs for rural patients increased by $29 \%$ compared to urban patients. In addition, costs increased by $23 \%$ for patients who did not follow the official care pathway. The extra costs associated with inappropriate referral pathway amount to int 172 (Table 1) indicating that indeed considerable costs might be saved implementing strategies to maintain official referral pathways. Inpatient care costs fluctuated over the last two years of the follow-up period, reflecting changes in the length of stay over time.

The results for the regressions on disease-specific annual inpatient costs per patient are presented in Table S8-S10 in the supplementary material. Across the three diagnoses, the factors that were consistently associated with higher inpatient costs were location, inappropriate referral pathways, older age, male gender and comorbidity. The effects on the costs of covariates were stronger among COPD patients as compared to stroke and IHD patients.

\section{Discussion}

Mean inpatient costs for patients diagnosed with COPD, IHD or stroke over the period 2016 to 2018 in Mongolia per patient per year was int 721 (sd=416), varying from int\$ 677 (sd=372) for official referral pathways to int 849 ( $s d=502)$ for unofficial referral pathways. Covariates significantly associated with high inpatient costs were location, age, gender and comorbidity. Our results show that costs per admission over three successive years went down in all hospital types when converting to the real price level in 2018. Although hospital funding increased in real terms, this growth was lower than the inflation rate, and explains the trends in int\$ term. Regarding disease-specific inpatient costs, part of the variation in inpatient costs may be explained by variation in the mean length of stay for all three diagnoses. Having comorbidity was associated with high inpatient costs for COPD patients, and less so for stroke and IHD patients. For all three diseases, the main inpatient cost drivers were location, inappropriate referral pathways, older age, and male gender, while social status had only a small effect on the inpatient costs.

Our study shows that referral pathways significantly affect inpatient costs and hence highlights the need for more efficient inpatient care delivery by stimulating the role of primary and secondary level hospital 
care, and enhancing official referral pathways. Similar pathways have been observed in other low- and middle-income countries, where unnecessary referrals lead to 1-2 month delayed diagnosis, ineffective treatment, and poor provider-patient communication for continued care support [21,22]. In countries with a large surface area like Mongolia, official referral systems will also reduce travel costs and productivity losses for patients and their families [10].

Comparing the costs currently estimated with previous findings in the literature was challenging due to the different perspectives used (societal or health care system), different cost components included (direct or indirect) and different phases of disease conditions studied. Keeping this heterogeneity of study designs in mind, internationally, a substantial variation in the mean inpatient costs was observed. For stroke patients, the mean annual inpatient costs per patient in China was int\$ 5264 for 27 bed-days in 2013 , including out-of-pocket payment (24.2\%) [23]. Another study conducted among patients hospitalized with acute coronary syndrome in eight Asian countries in 2013 found a mean length of stay of 10 days and per day cost in a critical unit that ranged from int \$3210 in Singapore, int\$ 284 in Thailand to int 98 in Vietnam [18]. For COPD patients, mean hospitalization costs were US\$3,670 in China [16] and US\$ 795 in Vietnam [17]. Our findings are in the low range of these numbers with mean costs of INT $\$ 722$ (sd=418), and cost per day below $\$ 100$. This is in line with per capita health spending from domestic resources which was US\$ 92 in Mongolia, but US\$ 63 in Vietnam, US\$ 250 in China, and US\$ 3500 in Japan [24]. Mean length of stay per patient was less than 10 days in our sample compared to a much longer mean length of stay in China (27 days) and Japan (30 days) for patient with stroke. In Mongolia, hospitals tend to assume that DRGs are providing funds for a standard 14 days of treatment. Therefore, current hospital payment mechanisms could be creating a shorter length of stay.

Our finding that age is positively associated with high inpatient costs is consistent with a study in eight Asian countries,[18] whereas a Chinese study found that younger stroke patients had higher inpatient costs than older patients due to better prevention programs [23]. Males had higher inpatient costs and longer length of stay compared to females, confirming findings in previous studies. Almost half of all men smoke and three-quarters consume alcohol compared to ten times fewer women smoking and two times fewer drinking alcohol [25]. Hence, the gender gap in health care use may largely be explained by exposure to modifiable risk factors such as tobacco us [26]. Referral to tertiary level hospitals is associated with significantly higher inpatient costs, supporting the findings from a Chinese study that showed mean inpatient costs in tertiary level hospitals were almost twice the amount of those in primary and secondary hospitals [15].

In rural areas, a total of 277 primary level hospitals were included in our study. Each have approximately 5-15 beds, realizing a total of 150 to 1300 admissions annually. In contrast, tertiary level general hospitals ( $n=3$ ) in the capital city, deliver 9500 to 25,000 specialized admissions annually. Ensuring equitable and accessible quality health care for all is the main challenge in the Mongolian health system's efforts to attain UHC, given the extremely low population density in a large part of its territory. Any unofficial policy decision and improper financial incentive can increase overall health expenditure and 
inefficient use of available resources in health, but also causes serious financial burdens on patients and families.

We were able to analyze the potential cost drivers for inpatient care use, investigating the role of patient characteristics and different treatment pathways. Findings represent 3 major NCDs and are based on patient-level data for a large number of hospitals and 3 years.

As with any analysis based on administrative data, our study lacked clinical information regarding the severity of disease. Therefore, we could not examine costs by severity level. Unit costs were estimated from public funding information using the payment methods applied for different health care providers, rather than activity-based expenditures information. Given that information from 303 hospitals had to be processed, that would have been an insurmountable task with little added value in terms of better estimates of per patient costs. We analyzed costs per patient per year funded by public resources rather than total inpatient costs due to the lack of financial information on OOP payment and health care use in private clinics.

Health care costs have increased over the years in response to demographic and epidemiological transitions under way in Mongolia (and other countries in Asia). To progress towards UHC, efficient use of available resources is crucial to controlling constantly increasing costs and growing demand. Previous local evidence found that public spending on NCDs in Mongolia was similar to NCDs spending observed in high-income countries and spending is dominated by inpatient care instead of preventive care. This apparently suggests inefficiency in resource use. To support future policy development, our findings add more detailed patient-level data while revealing that inpatient costs associated with unofficial referral pathways were significantly higher. Policy reform to address the latter would free resources that could be used toward achieving universal health coverage. Various policy efforts can ensure more efficient referral pathways through providing alternative care for patients, altering incentives to care providers, and raising knowledge about efficient admission practice. Other policy options are better coordination and control and official NCD disease management. In sum, it would appear that there is ample room for eliminating unnecessary spending on hospital care. The fact that some of the direct referrals might have been appropriate from a clinical point of view cannot be ruled out. We feel, however, this might be the case in a minor proportion only.

Also, Mongolia has massive opportunities to benefit from proactive prevention and health promoting activities. According to the national survey, one in every three Mongolians has three or more common modifiable risks factors for developing NCDs [25]. In absence of strategic prevention programs, the costs associated with preventable NCDs will continue to grow, resulting in an increased demand for inpatient care. This on top of the demographic trend of an ageing population may result in a non-sustainable financial burden to the patient, health system and society [27]. Therefore, action should be considered to implement cost-effective prevention focusing on common NCD risk-factors. The time is now, since $65 \%$ of the population is still under the age of 35 . 
Our findings may inform policy makers on the trends in NCDs and their associated costs, and could provide useful information to inform future cost-effectiveness studies of disease prevention and health promotion programs related to the three major NCDs in Mongolia and comparable LMICs.

\section{Conclusion}

The inpatient costs for major NCDs were substantially lower when an official referral pathway was followed and a substantial proportion of the inpatient care use consisted of pathways that may indicate unofficial self-referrals in Mongolia. Reducing the unofficial use of inpatient care can improve efficient resource allocation in health care and prevent further escalation of inpatient costs in the future.

Additionally, this study supports further research into the cost-effectiveness of prevention in order to shift costs from reactive hospital care to prevention policies in Mongolia as well as other developing countries.

\section{Declarations}

\section{Acknowledgements}

The authors would like to acknowledge the medical information specialists at the Health Development Center in Mongolia, for their assistance with data extraction. We also would like to thank Steef Konings from the University of Groningen for his help in analysing the data.

\section{Funding}

No funding was received for conducting the study or for writing this manuscript.

\section{Conflict of interest}

The authors declare that there are no conflicts of interest.

\section{Availability of data and material}

The datasets generated during and/or analysed during the current study are available from the corresponding author on reasonable request.

\section{Ethical approval}

This study was approved by the Center for Health Development in Mongolia according to their guidelines.

\section{References}


[1] C. E. Bloom DE, JanéLlopis E, Abrahams-Gessel S, Bloom LR, Fathima S, Feigl AB, Gaziano T, Hamandi A, Mowafi M, O'Farrell D., "The global economic burden of noncommunicable diseases," vol. The global economic burden of noncommunicable diseases., 2012. [Online]. Available: https://ideas.repec.org/p/gdm/wpaper/8712.html.

[2] T. Muka et al., "The global impact of non-communicable diseases on healthcare spending and national income: a systematic review," Eur J Epidemiol, vol. 30, no. 4, pp. 251-77, Apr 2015, doi: 10.1007/s10654-014-9984-2.

[3] C. D. Mathers and D. Loncar, "Projections of global mortality and burden of disease from 2002 to 2030," PLoS Med, vol. 3, no. 11, p. e442, Nov 2006, doi: 10.1371/journal.pmed.0030442.

[4] WHO, "Global spending on health: a world in transition," World Health Organization, no. (No. WHO/HIS/HGF/HFWorkingPaper/19.4), 2019.

[5] S.-O. E. Tsilaajav T, Baasai B, Byambaa G, Shagdarsuren O. , "Mongolia Health System Review. Vol.3 No.2. Manila: World Health Organization, Regional Office for the Western Pacific, ," 2013.

[6] Vasoontara Y, Judith Healy, and H. Kendig, "Health system responses to population ageing and noncommunicable diseases in Asia," 2016.

[7] WHO, "The world health report: health systems financing: the path to universal coverage," 2012.

[8] " The World Bank's classification of countries by income. The World Bank; 2019-2020."

[9] "Mongolia Population (2019) - Worldometers [Internet]. Available from:

https://www.worldometers.info/world-population/mongolia-population/."

[10] J. Dorjdagva, E. Batbaatar, M. Svensson, B. Dorjsuren, B. Batmunkh, and J. Kauhanen, "Free and universal, but unequal utilization of primary health care in the rural and urban areas of Mongolia," Int $J$ Equity Health, vol. 16, no. 1, p. 73, May 8 2017, doi: 10.1186/s12939-017-0572-4.

[11] D. Bayarsaikhan, S. Kwon, and D. Chimeddagva, "Social health insurance development in Mongolia: Opportunities and challenges in moving towards Universal Health Coverage," (in English), International Social Security Review, vol. 68, no. 4, pp. 93-113, Oct-Dec 2015, doi: 10.1111/issr.12089.

[12] O. Dugee, E. Munaa, A. Sakhiya, and A. Mahal, "Mongolia's Public Spending On Noncommunicable Diseases Is Similar To The Spending Of Higher-Income Countries," Health Aff (Millwood), vol. 36, no. 5, pp. 918-925, May 1 2017, doi: 10.1377/hlthaff.2016.0711.

[13] MoH, " Health Indicators The Centre for Health Development; 2018. Available: http://www.chd.mohs.mn.," 2018. 
[14] WHO, "Health at a Glance: Asia/Pacific 2018 MEASURING PROGRESS TOWARDS UNIVERSAL HEALTH COVERAGE," 2018.

[15] S. Hu et al., "Economic Burden of Individual Suffering from Atrial Fibrillation-Related Stroke in China," Value Health Reg Issues, vol. 2, no. 1, pp. 135-140, May 2013, doi: 10.1016/j.vhri.2013.02.008.

[16] M. Li et al., "Factors contributing to hospitalization costs for patients with COPD in China: a retrospective analysis of medical record data," (in English), International journal of chronic obstructive pulmonary disease, vol. 13, pp. 3349-3357, 2018.

[17] C. Q. Ngo et al., "Direct Hospitalization Cost of Patients with Acute Exacerbation of Chronic Obstructive Pulmonary Disease in Vietnam," Int J Environ Res Public Health, vol. 16, no. 1, Dec 302018 , doi: 10.3390/ijerph16010088.

[18] S. Jan et al., "Predictors of high-cost hospitalization in the treatment of acute coronary syndrome in Asia: findings from EPICOR Asia," BMC Cardiovasc Disord, vol. 18, no. 1, p. 139, Jul 4 2018, doi: 10.1186/s12872-018-0859-4.

[19] T. Bolormaa, Natsagdorj, Ts, Tumurbat, B, Bujln, Ts, Bulganchlmeg, B. et al., "Health systems in transition : Mongolia," WHO Regional Office for Europe, 2007.

[20] W. Bank, "International Comparison Program: World Development Indicators database," Eurostat-OECD PPP Programme, 2019.

[21] J. Goudge, L. Gilson, S. Russell, T. Gumede, and A. Mills, "Affordability, availability and acceptability barriers to health care for the chronically ill: Iongitudinal case studies from South Africa," BMC Health Serv Res, vol. 9, p. 75, May 9 2009, doi: 10.1186/1472-6963-9-75.

[22] V. Yiengprugsawan, Healy, J. and Kendig, H., "Health system responses to population ageing and noncommunicable diseases in Asia.," 2016.

[23] H. Zhang, Y. Yin, C. Zhang, and D. Zhang, "Costs of hospitalization for stroke from two urban health insurance claims data in Guangzhou City, southern China," (in English), BMC health services research, vol. 19, no. 1, p. 671, 2019.

[24] "Global Health Expenditure Database, World Health Organization," doi: https://apps.who.int/nha/database/country_profile/Index/en.

[25] WHO, "Third national STEPS Survey on the Prevalence of Noncommunicable Disease and Injury Risk Factors," 2013.

[26] A. Tuvdendorj, T. Feenstra, B. Tseveen, and E. Buskens, "Smoking-attributable burden of lung cancer in Mongolia a data synthesis study on differences between men and women," PLoS One, vol. 15, no. 2, p. e0229090, 2020, doi: 10.1371/journal.pone.0229090. 
[27] D. Bayarsaikhan and K. Nakamura, "Health promotion financing with Mongolia's social health insurance," Asia Pac J Public Health, vol. 27, no. 2, pp. NP887-96, Mar 2015, doi: 10.1177/1010539509332449.

\section{Tables}

Table 1 Distribution of inpatient costs stratified by type of referral pathway 


\begin{tabular}{|c|c|c|c|c|c|c|c|}
\hline \multirow[t]{3}{*}{ Variables } & \multirow{2}{*}{\multicolumn{2}{|c|}{$\begin{array}{l}\text { Overall } \\
N=117,623\end{array}$}} & \multirow{2}{*}{\multicolumn{2}{|c|}{$\begin{array}{l}\text { Yes/official } \\
N=87,260\end{array}$}} & \multirow{2}{*}{\multicolumn{2}{|c|}{$\begin{array}{l}\text { No/unofficial } \\
N=30,363\end{array}$}} & \multirow[t]{3}{*}{$P$ value } \\
\hline & & & & & & & \\
\hline & mean & sd & mean & sd & mean & sd & \\
\hline Mean costs in int\$ & 721 & $(416)$ & 677 & $(372)$ & 849 & $(502)$ & \\
\hline Gender & & & & & & & NS \\
\hline Male & 739 & $(444)$ & 690 & $(396)$ & 862 & $(528)$ & \\
\hline Female & 708 & (395) & 667 & $(352)$ & 837 & $(476)$ & \\
\hline \multicolumn{8}{|l|}{ Age group } \\
\hline $0-40$ & 668 & $(370)$ & 622 & $(306)$ & 820 & $(489)$ & 0.00 \\
\hline $40-50$ & 730 & $(434)$ & 680 & $(368)$ & 877 & $(546)$ & \\
\hline $50-60$ & 718 & $(422)$ & 676 & $(378)$ & 842 & $(510)$ & \\
\hline $60-70$ & 723 & $(424)$ & 679 & $(384)$ & 836 & $(495)$ & \\
\hline $70-80$ & 748 & $(415)$ & 705 & $(383)$ & 860 & $(471)$ & \\
\hline $80+$ & 744 & $(418)$ & 699 & $(384)$ & 876 & $(480)$ & \\
\hline \multicolumn{8}{|l|}{ Location } \\
\hline Rural & 787 & $(435)$ & 751 & $(386)$ & 889 & $(532)$ & 0.00 \\
\hline Urban & 601 & $(355)$ & 541 & $(301)$ & 774 & $(432)$ & \\
\hline \multicolumn{8}{|l|}{ Comorbidity } \\
\hline 0 & 717 & $(406)$ & 675 & $(361)$ & 840 & $(497)$ & 0.04 \\
\hline $1-2$ & 725 & $(425)$ & 679 & $(379)$ & 855 & $(506)$ & \\
\hline \multicolumn{8}{|l|}{ Social status } \\
\hline Formal sector & 711 & $(389)$ & 667 & $(329)$ & 836 & $(504)$ & 0.00 \\
\hline Private sector & 688 & (393) & 648 & $(338)$ & 861 & $(535)$ & \\
\hline Herders & 796 & $(432)$ & 757 & $(382)$ & 885 & $(517)$ & \\
\hline Pensioners & 727 & $(419)$ & 684 & $(384)$ & 842 & $(484)$ & \\
\hline Unemployed & 702 & $(421)$ & 653 & $(357)$ & 859 & $(528)$ & \\
\hline Others & 702 & $(424)$ & 660 & $(374)$ & 854 & $(541)$ & \\
\hline
\end{tabular}

$\mathrm{SD}=$ standard deviation 
Table 2 Multivariate analysis of annual inpatient costs per patient for three DRGs

(Generalized linear model, log-link link with clustered standard errors, price level at 2018) 


\begin{tabular}{|c|c|c|}
\hline \multirow[t]{2}{*}{ Variables } & Coefficient & \multirow[t]{2}{*}{ Clustered SE } \\
\hline & (exponential) & \\
\hline Observations & 117,623 & \\
\hline \multicolumn{3}{|l|}{ Intercept } \\
\hline int\$ & 642.2 & $(0.009)$ \\
\hline \multicolumn{3}{|c|}{ Gender (ref=female) } \\
\hline Male & $1.04^{\star \star \star}$ & $(0.004)$ \\
\hline \multicolumn{3}{|c|}{ Age group $(r e f=0-40)$} \\
\hline $40-50$ & $1.08^{\star \star \star}$ & $(0.007)$ \\
\hline $50-60$ & $1.09 * \star \star$ & $(0.006)$ \\
\hline $60-70$ & $1.10 * \star \star$ & $(0.008)$ \\
\hline $70-80$ & $1.14^{\star \star \star}$ & $(0.009)$ \\
\hline $80+$ & $1.15^{\star \star \star}$ & $(0.011)$ \\
\hline \multicolumn{3}{|c|}{ Location (ref=urban) } \\
\hline Rural & $1.29 * \star \star$ & $(0.004)$ \\
\hline \multicolumn{3}{|c|}{$\begin{array}{l}\text { Social status } \\
\text { ref=Formal sector) }\end{array}$} \\
\hline Private sector & $0.98^{\star \star}$ & $(0.009)$ \\
\hline Herders & 1.01 & $(0.009)$ \\
\hline Pensioners & $0.98 * *$ & $(0.009)$ \\
\hline Others & $0.98 * *$ & $(0.008)$ \\
\hline \multicolumn{3}{|c|}{ Comorbidities $(r e f=0)$} \\
\hline $1-2$ & $1.01^{\star}$ & $(0.004)$ \\
\hline \multicolumn{3}{|c|}{ Official referral pathways (ref=Yes) } \\
\hline No & $1.23^{\star \star \star}$ & $(0.005)$ \\
\hline \multicolumn{3}{|c|}{ Year (ref=2016) } \\
\hline 2017 & 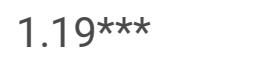 & $(0.004)$ \\
\hline 2018 & $0.91^{\star \star \star}$ & $(0.004)$ \\
\hline
\end{tabular}

${ }^{*} p<0.1 ;{ }^{* \star} p<0.05 ;{ }^{* \star *} p<0.01$ 


\section{Figures}

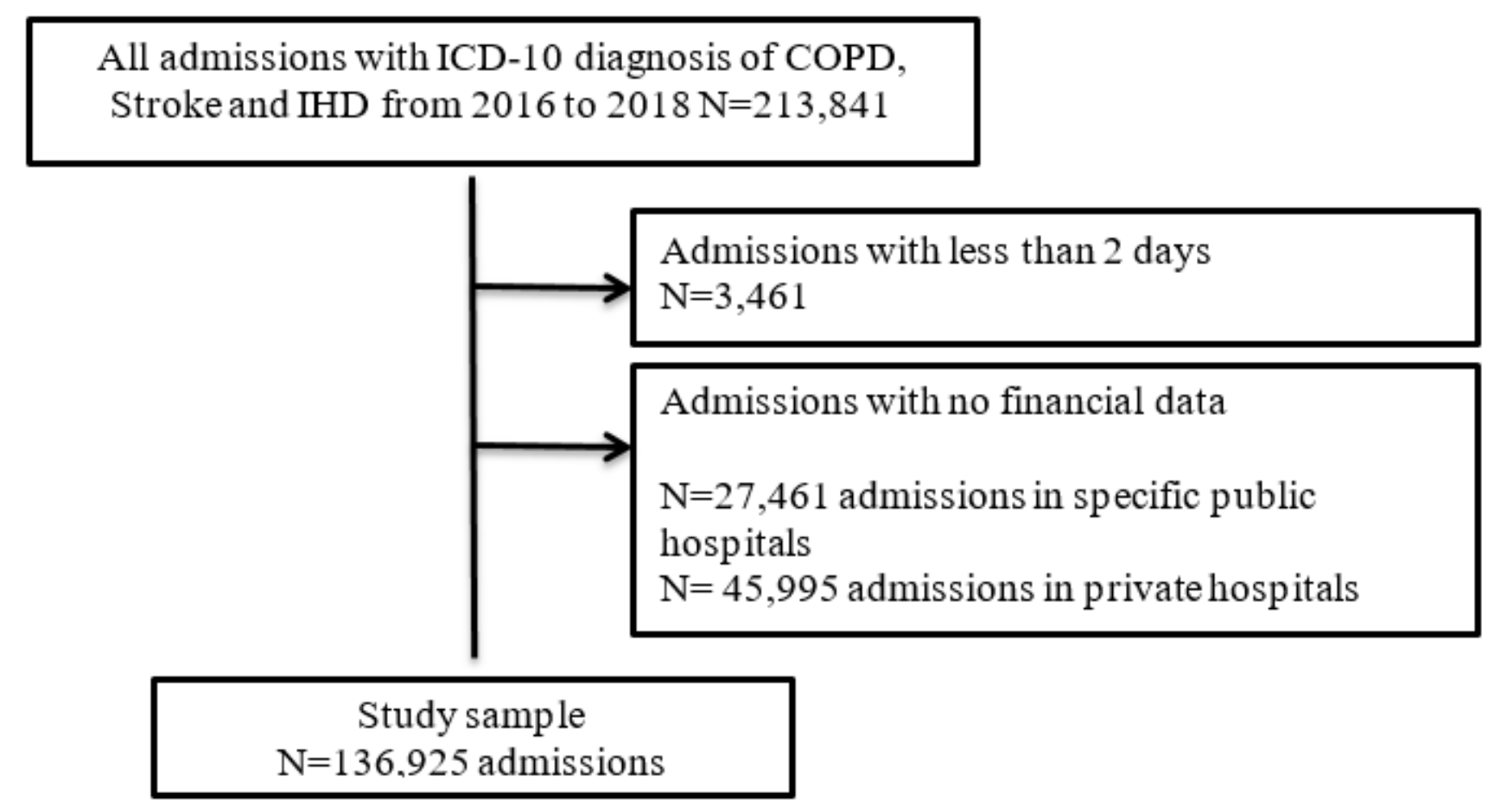

Figure 1

Selection criteria for study sample 

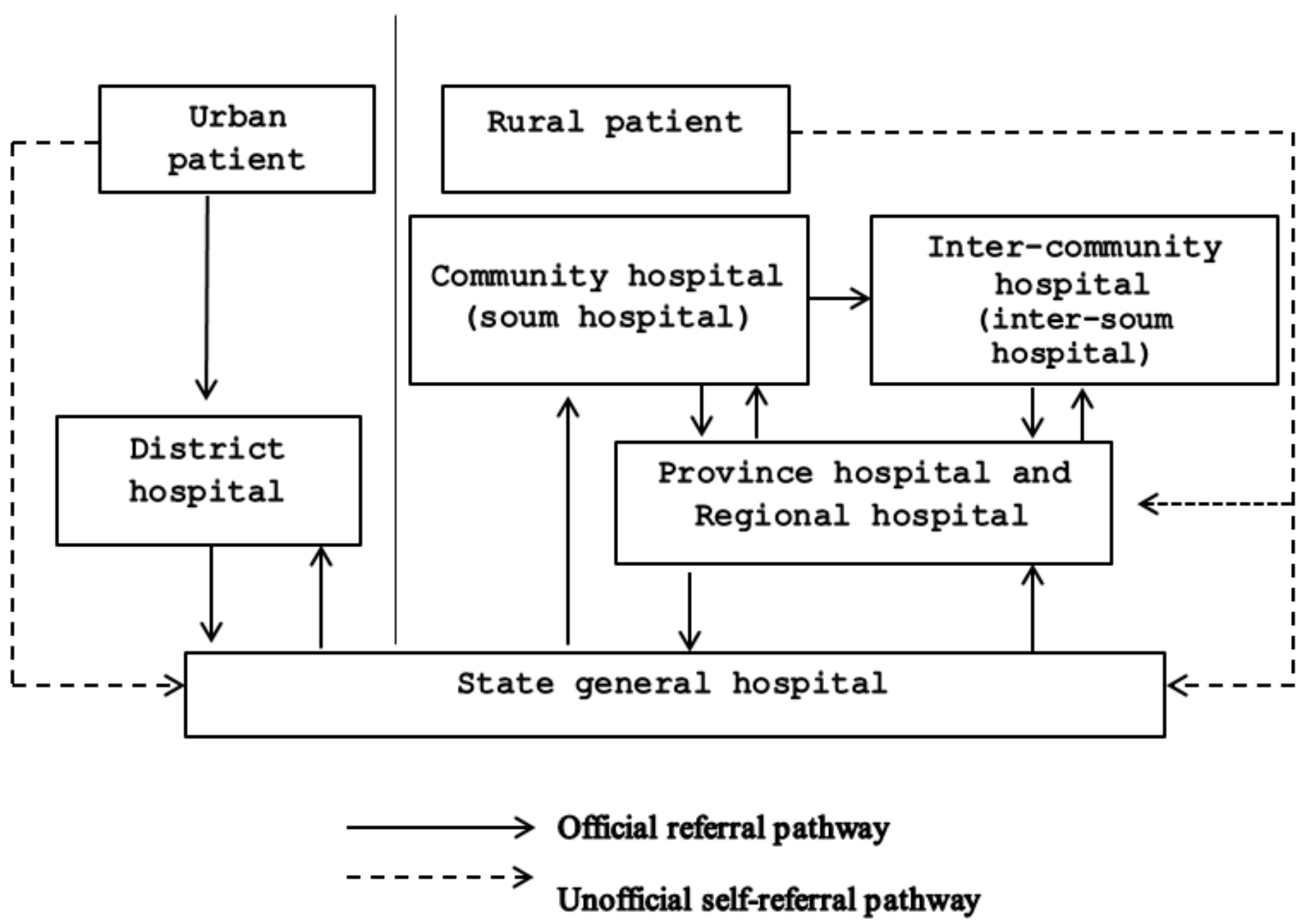

Figure 2

Referral pathway
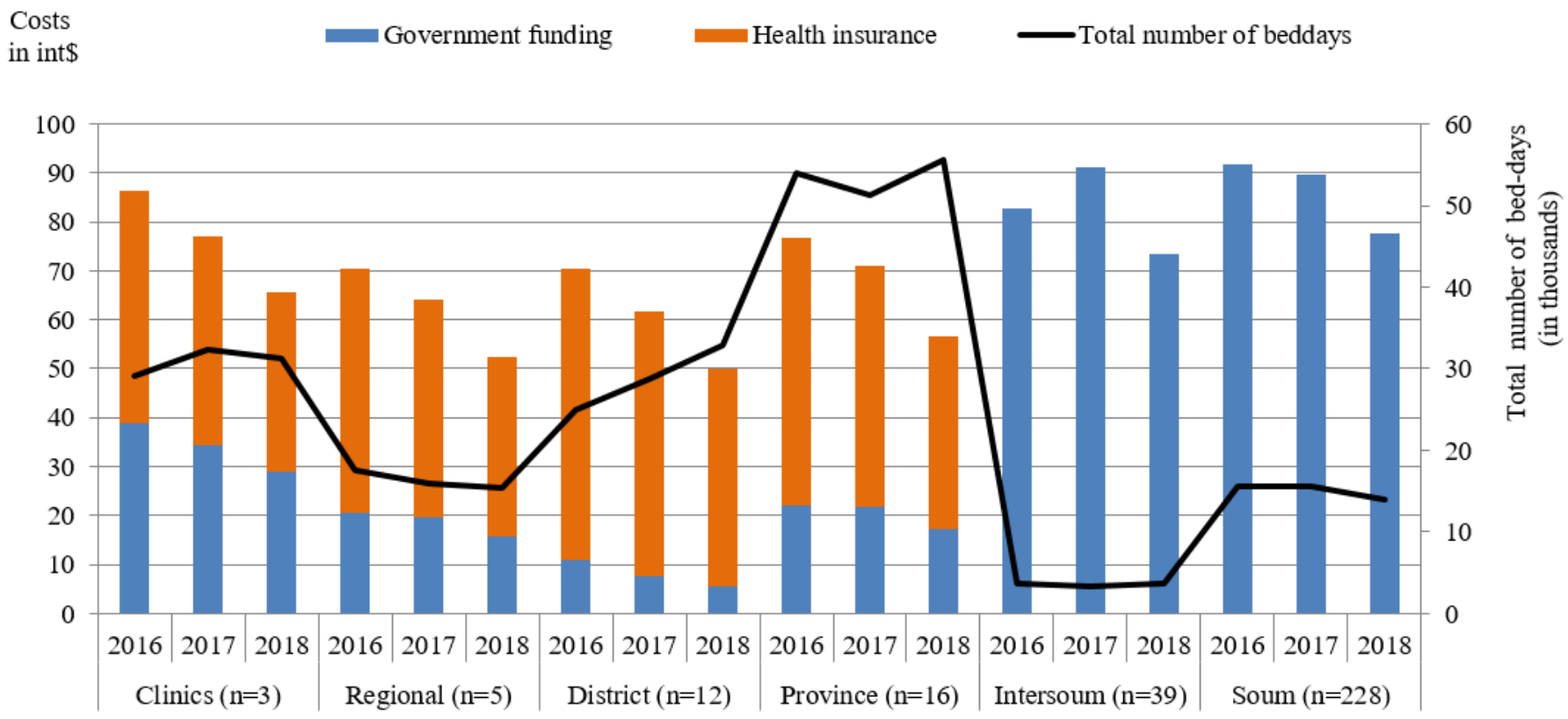

Type of hospital 
Figure 3

Cost per bed-day by types of hospital and years for stroke, in int\$ 2016-2018.

\section{Supplementary Files}

This is a list of supplementary files associated with this preprint. Click to download.

- SupplementaryBMC.docx 